\title{
A disaster prevention programme using virtual schemes: Recommendation of tradition populaire integrated with tendenko as an approach to immersive training
}

\author{
Hiroko $\mathrm{Oe}^{1}$ and Sachiyo Kawakami ${ }^{2}$ \\ ${ }^{1}$ Bournemouth University \\ hoe@bournemouth.ac.uk \\ ${ }^{2}$ University of Fukui \\ sachi-k@u-fukui.ac.jp
}

\begin{abstract}
The aim of this technical note is to discuss and propose a community action plan to increase citizen preparedness and readiness for natural disasters. This exploratory study applies a conceptual analysis based on a comprehensive review of relevant literature on evacuation training and preparation for tsunamis, focusing on tendenko behaviour.

The results of the study led to the development of a guideline outlining key actions for evacuation programme designers with practical procedures to foster citizens' motivation to learn and encourage engagement in community-based activities. The proposed steps can provide training designers (e.g. local councils, community NPOs, and evacuation experts) with actionable recommendations for practical activities to enhance citizen preparedness.
\end{abstract}

One of the original contributions of the proposed programme is its inclusion of tradition populaire, according to which communication is held not only with the residents in the disaster area but also with inbound visitors. The proposed disaster prevention training programme based on information and communications technology (ICT) can be supportive to the disaster areas by inbound visitors to enhancing citizens' and visitors' 
understanding of disastrous scenarios and the necessity to increase their preparedness for such events.

Based on the outcomes of the research, a further analysis is being planned to validate the utility and effectiveness of the proposed action plans, with primary data being used to develop more robust implications for practitioners and researchers.

Keywords: tendenko, preparedness, natural disasters, AR/VR, immersion, tradition populaire, learning programme

\section{Introduction}

\subsection{Background of the study: Japanese experiences}

'Disaster preparedness refers to measures taken to prepare for and reduce the effects of disasters. That is, to predict and, where possible, prevent disasters, mitigate their impact on vulnerable populations, and respond to and effectively cope with their consequences' (The International Federation of Red Cross and Red Crescent).

Disaster prevention drills, including tendenko training, should be a standard measure to increase citizens' preparedness for disasters and reduce the loss sustained from potential future disaster events (Tsuneyoshi, 2019)[1]. Magnussen et al. (2018)[2] has also confirmed that collaborative exercises among a community increase awareness and enhance prevention activities. 
Sun et al. (2013)[3] has discussed tsunami-tendenko as one of the critical methods that citizens should follow in the event of a tsunami. However, it is known there are a few key psychological barriers to following this rule. As a method, Tendenko adheres to an 'everyone for him/herself' mindset, calling for individual evaluations of actions that do not consider other people, even family members.

During the Great East Japan Earthquake in 2011, this approach worked to mitigate the number of the victimised citizens. However, it was not easy for residents to follow, as they felt guilt based on moral and emotional obstacles (Hatayama and Nakai, 2020)[4]. So far, various methods for increasing citizens' preparedness for forthcoming disasters have been discussed, and a number of issues have been addressed by regional agendas to provide safe infrastructure and prevention training programmes based on a community framework.

\subsection{Research gap}

Although the utility of preventive training programmes in communities to increase citizens' preparedness for natural disasters has been discussed among practitioners and researchers, actionable implications with practical recommendations have not yet been developed. In the field of prevention training drills, systematic approaches have lacked depth, resulting in a scarcity of practical guidance for relevant researchers and practitioners (Hangartner et al., 2019)[5].

Recently, a focus on information and communications technology (ICT) measures to provide virtual training platforms have been discussed. However, holistic recommendations on how to integrate preventive activities and virtual learning materials 
should be explored in greater depth so that community training designers can develop effective programmes responding to community needs and experiences.

\subsection{Aim and objectives}

This study aims to discuss and propose a holistic training programme for increasing citizens' preparedness for natural disasters. It is suggested that virtual learning modules are implemented in communities to enhance citizen preparedness based on nurturing the mindset of tendenko, which has been acknowledged as a useful strategy in the case of tsunamis. To achieve this aim, three objectives were developed:

1) To review relevant academic discussions and draw key lessons in the context of tendenko behaviour and other Japanese experiences from the Great East Japan Earthquake in 2011

2) To discuss action plans along with key themes on how to enhance citizens' preparedness for disasters so as to reduce the number of natural disaster victims

3) To discuss and propose a preventative training programme framework containing key factors that should be included in all such programmes and to present a further research plan

\section{Academic discussion}

\subsection{Natural disasters and tendenko}

Tsuneyoshi (2019)[1] discusses safety and disaster prevention programmes from an educational point of view. In these discussions, it is proposed that tendenko behaviour leads to ideal actions during disaster incidents. As noted, the mindset of tendenko has been widely acknowledged and recommended, especially for tsunami incidents. However, it is 
also well known that there are psychological and ethical barriers to adopting such a mindset (Sun et al, 2013)[3]

Goltz (2017)[7] examines the concept of tendenko from a disciplinary perspective, clearly defining it as 'a tsunami evacuation strategy that advocates a rapid individual response to an imminent tsunami threat by moving quickly to safety, disregarding others, even one's own family members' (Goltz, 2017:1)[7]. As discussed, the concept of tendenko was developed partly within the field of disaster prevention. It also stemmed from folklore, or tradition populaire, nurtured in the eastern coast of Japan, where people have experienced significant earthquakes and tsunamis for centuries.

As Thompson (2019)[8] has discussed, high death tolls during tsunamis have been attributed to family members being eager to reunite while evacuating, this leading to fatalities because they missed the critical moment of evacuation. In the Great East Japan Earthquake that took place on March 11, 2011, it was found that the execution of tendenko saved the lives of many people. After this experience, the concept of tendenko emerged as a theme to be acknowledged in the event of a disaster (Tsuneyoshi 2019)[1]. Goltz (2017)[7] examines two related points and recommends that tendenko can be facilitated by the human tendency to act individually rather than collectively in a crisis situation, and that practicing tsunami tendenko will save lives. In line with such discussions, the significance of tendenko training in increasing preparedness for tsunami disasters has been agreed upon by many scholars (e.g., Kawakami et al., 2020; Kodama, 2015; Maruyama, 2018)[9][10][11].

The 'Miracle of Kamaishi' is a well-known event in Japan (Enshu, 2014)[12], and the experience of Kamaishi City, Iwate Prefecture, during this event is noteworthy. The city is a small one, with a population of 40,000 , and during one significant tsunami more 
than 1,000 people were killed, meaning 1 in 40 citizens died. However, in this particular case, almost all 2,000 elementary and junior high school students in the city escaped the tsunami and survived. Unfortunately, the number of victims was not zero, with five children being killed. Still, based on the number of victims throughout the city, it was estimated that nearly 80 children could have been killed (Kamaishi City, 2016)[13]. As Enshu (2014)[12] emphasises, the 'Miracle of Kamaishi city' was based on tendenko behaviour, and it should be celebrated that only five children died, as this was a phenomenal result. Further, it is evidence of the achievement of the local disaster prevention programme based on the slogans 'tsunami tendenko' and 'life tendenko'.

In line with this discussion, Maeda and Hashimoto (2020)[14] note that The Great East Japan Earthquake was a reminder of the importance of prevention training, the memory of which had grown hazy since the Great Hanshin-Awaji Earthquake in January, 1995. Disaster prevention education whose main purposes are 'self-help' and/or 'mutual help' have saved many lives. The Ministry of Education, Culture, Sports, Science and Technology (2013)[15] has also note that, as natural disasters are difficult to predict, disaster prevention education at schools is critical to increase preparedness. In such cases, tendenko behaviour has been a central concept supporting the prevention training.

\subsection{Potential of virtual learning systems to increase citizens' preparedness}

To instil tendenko and other risk mitigation behaviours, continuous training and drills should be planned. In the community learning process, a supportive IT-based scheme could be useful in enhancing the awareness of potential risks and preparedness for disasters (Oe \& Yamaoka, 2020)[16]. To instil the meaning of tendenko among citizens in communities, sharing information through hands-on training is essential (Hatayama \& 
Nakai, 2018)[4]. In doing so, an e-learning system within communities could contribute to deepening citizens' understanding of tendenko both critically and virtually. Using the system, citizens would review and practice mitigation behaviours in preparing for natural disaster incidents (Mikami et al., 2018)[17]. At the same time, it is not easy to use ITbased schemes, such as e-learning systems, to achieve the stated goal (preparedness in this context), and it has been acknowledged that there are several gaps that need filling in this regard (Paton, 2019)[18].

\subsection{How to fill the gap between reality and possible future disasters in training}

To develop the research questions, Figure 1 presents an analytical framework for the study. In particular, it outlines how to fill the perception gap and reduce panic and fear to enable citizens to behave in a protective manner while responding to natural disasters. The framework also presents key topics on the theme of how virtual learning platforms can enhance citizens' preparedness for disasters.

Figure 1. Virtual and real-word perception gap model (Inspired by Mitsuhara (2018)[19] and arranged by the authors)

Further, it should be required that we focus on better-integrated prevention programs with the ability to enhance citizens' preparedness for natural disasters. In other words, there exists the critical issue of how to improve existing disaster prevention programs to support prevention behaviour. As a country with many natural disasters, including huge earthquakes and tsunamis (such as the expected Nankai Trough Earthquake), there have been many experiences in and attempts to provide well- 
developed disaster preventing training programs, and many have been discussed and implemented (Yanagawa et al., 2016)[20]. At the same time, it has been acknowledged that it is not easy to maintain citizens' consciousness in preparing for expected but not forecasted future disasters (Guo \& $\mathrm{Li}, 2016)[21]$. To fill this vacancy of consciousness and sustain awareness in citizens by shortening the gap of perceptions towards future disasters, e-learning systems could integrate the key points of tendenko with real examples and visualized materials on a virtual basis in a practical and effective prevention training programme (Palen \& Liu, 2007)[22].

\subsection{Community-based programme: tradition populaire}

One suggestion that can be derived from the idea of a community-based approach is to draw inspiration from folklore and pay attention not only to the residents in the disaster area but also inbound visitors. The proposed disaster prevention training programme based on ICT measures will have the ability to enhance citizens' understanding of the importance of tendenko as a code of practice by enabling them to experience tsunami disasters through virtual measures. At the same time, the proposed e-learning programme could be an enlightening attraction to visitors and increase their awareness of the necessity of disaster prevention behaviour based on tendenko.

Historically, various methods have been used to pass down experiences and knowledge concerning natural disasters based on tradition populaire. For example, the Kobe Luminarie, which is held at the end of each year, is used to pass down the tragedy of the Great Hanshin-Awaji Earthquake of 1995, and it has become a tradition of the Kobe to mourn the dead during their festival. At such festivals, it is also important to remember the sadness of these events. In addition, the areas affected by the Great East Japan 
Earthquake have been tsunami-prone since ancient times. In these areas, the idea that when a tsunami hits you should first escape to a hill to save yourself (tsunami-tendenko) has been taken up in stories in recent years.

More recently, the use of new digital technologies, such as augmented reality (AR) and virtual reality (VR), has been able to provide citizens with experiences of immersion through virtual platforms. Skinner (2018)[23] suggests that the implementation of AR/VR and animation could enable citizens to learn about and understand disaster impacts and increase their preparedness using sensory experiences. This approach could also be discussed in line with the sub-effects of dark tourism (Stone, 2013)[24]. Hooper and Lennon (2016)[25] claim that the concept of dark tourism should not be based on the visiting of tragic ruins from the point of view of curiosity but instead on recalling and reviewing tragic incidents so that visitors can increase their disaster preparedness, thus saving lives based on acquired knowledge and virtual experiences.

Increasing awareness and enhancing learning opportunities by increasing mental resilience and preparedness are critical in prevention training to respond to future disasters. For instance, '3.11 Tradition Road' was established as an NGO to register and convey lessons learned from the Great East Japan Earthquake, which are expected to lead to preparations aimed at disaster prevention (Denshoroad organisation, 2019)[26]. It has been discussed that there are two pillars contributing to the improvement of disaster prevention capabilities: (1) handing down lessons learned from the Great East Japan Earthquake and (2) contributing to regional revitalisation through interaction with a large number of visitors (e.g. establishing maps, organizing model routes, and planning traditional tours; Sasaki et al., 2018)[27]. 


\section{Discussion, conceptual map proposition, and plans for future work}

\subsection{Perspectives for the training design}

In the context of risk management for natural disasters, communities should be redesigned as 'learning organisations' with enhanced learning tools, including ICT-based ones, to increase preparedness. The use of an e-learning system in communities to support citizens' preparedness for natural disasters should be investigated to cover a scope different from that of the subjects taught at schools. It is critical to use e-learning to fill the psychological gap and instil lessons leading to the real behavioural change that is required in the actual disaster incidents.

As Oe (2019a)[28] has discussed, natural disasters have revealed an information gap issue, especially for vulnerable citizens, and this requires researchers to explore how to support disabled people with ICT applications. Further, designing effective learning processes should be discussed based on a holistic view of how to build sustainable communities, inviting various relevant stakeholders to support the communities hit by disasters. Oe (2011a)[29] also suggests a training menu using an etude method based on the case lead approach.

The concept of immersion through technological development is also applicable as a key theme for disaster preparedness training. This has been previously discussed in relation to the emergence of virtual environments based on the scientific innovation of technology (Lovreglio \& Kinateder, 2020)[30]. In particular, Scientists have explained that the immersion is due mostly to the development of appreciative criteria for threedimensional (3D) games, according to which players can fully experience the reality of the virtual worlds through surround sound and realistic interactive 3D environments (Macchione et al. 2019)[31]. However, the concept of immersion in designing disaster 
prevention/learning programmes has not been thoroughly researched, and the development of computer-generated imagery represents an opportunity to enhance immersion in learning experiences based on virtual learning (Solinska-Nowak et al., 2018)[32]. An innovative programme based on the concept of immersion and using continuous reviewing could enhance citizens' preparedness, increasing their awareness, deepening their understanding, and embodying the virtual learning outcome in a real disaster context. A conceptual model of this idea is presented in Figure 2.

Figure 2. Steps to enhance preparedness and readiness based on e-learning training (Inspired by Scharmer (2017)[33] and arranged by the authors)

As technical pathways with actionable recommendations, Figure 3 shows five sub-topics for the action plan with key elements from Japanese experiences based on recommendations from traditional populaire. These are to be implemented using a virtual learning platform, such as AR/VR, in the context of an immersion approach to disaster prevention.

Figure 3. Action plan for five sources to design immersion programme based on tradition populaire and tendenko (authors' collection)

\subsection{Future research agenda}

Japan is a country of natural disasters, and e-learning systems can be used to enhance the links between learning platforms and protect citizens within the region. E-learning systems 
further represent action-oriented learning platforms that could increase collaborative preparedness towards natural disasters.

The preparation of effective prevention training schemes has been on the agendas of local councils in Japan for decades, especially after the Great East Earthquake in 2011 (Oe, 2011b)[34]). Oe (2011b)[34] also emphasises the critical point of risk communication, which should be supported by interactive relationships among learningcommunity members.

Last but not least, a standardised learning platform could be used on a wide scale, meaning lessons for disaster prevention could be included in designing a plan for an entire city $(\mathrm{Oe}, 2019 b)[35]$.

\subsubsection{Approach and samples}

Based on this research study as a pilot exploration, the next step will be conducted based on a qualitative approach to examine the proposed framework and develop actionable implications for the implementation of risk management and disaster prevention training. As the aim of the research is to investigate and evaluate the perceptions of citizens towards disaster drills and practices, the best-placed research method involves a qualitative approach to explore the data (Tetnowski, 2015)[36]. Using a semi-structured questionnaire designed based on the literature review, interviews will be conducted with three groups of participants: (1) local council officials (planners of prevention education schemes), (2) citizens (e-learners of the prevention training), and (3) local experts and advisors. The authors will employ the convenience and snowball sampling methods (Edmonds, 2019)[37] to the initial participant, who will be asked to recommend acquaintances who might be happy to contribute. Following this process, active 
participants in prevention training among communities, local council officials, and experts and advisors will be invited to participate in the study.

A combination of various opinions and thoughts from three groups of people will help to triangulate the data and enhance its credibility (Jentoft and Olsen, 2019)[38]. Triangulation can also help in increasing the validity of the inferences made in qualitative research, as articulated by Kern (2018)[39]. The method will also assist in controlling for biases and fulfilling the stated objectives of the study.

The paper will also advocate for the incorporation of tendenko in a preventive training programme for communities vulnerable to tsunamis. Thus, to achieve the future aim of the research, specific communities have been identified in which this study will likely be carried out, namely, Minato-ku, Central Tokyo, and the Tokatsu area of Chiba Prefecture. Both areas suffered serious earthquakes and tsunami threats during the incident in 2011.

\subsubsection{Data analysis plan}

The interviews will be recorded and transcribed, and the obtained data will be analysed using the text mining software NVivo 11 to develop key themes and contexts concerning how to use and design a prevention training programme (Auerbach \& Silverstein, 2003)[40]. A thematic analysis will also be conducted on the obtained data.

\section{Conclusion}

\subsection{Contribution}

The established propositions were derived from existing theories based on an inductive approach. In designing a community-based prevention programme, the conceptual framework (Figure 2) and five proposed factors that should be included in the learning 
programme (Figure 3) can be further enhanced to suit various contexts. The proposed conceptual framework and action plan are a generic model, providing steps to enhance citizens' preparedness and readiness for future natural disasters. The framework can be used to develop standard prevention training programmes with practical procedures to foster citizens' learning outcomes and encourage citizen engagement in the training programmes.

At the same time, the proposed steps can provide training designers, such as local council officials, with practical guidance on how to embed local assets and memories to support learning outcomes. The framework suggests that collaborative relationships between citizens and programme managers is critical.

\subsection{Original contributions}

This research remains a pilot discussion with the tentative outcome of an exploration into a community-based evacuation programme aimed at preparing for natural disasters. In the field of prevention training, previous systematic approaches have lacked a depth, resulting in a lack of conceptual frameworks agreed upon as solid guidance for relevant researchers and practitioners.

The outcomes of this study are the development of a guideline with key actions for evacuation programme designers and practical procedures to foster citizens' motivation to learn and encourage citizen engagement in community-based activities. The proposed steps can provide training designers (e.g. local councils, community NPOs, and evacuation experts) with actionable recommendations and practical activities to enhance citizen preparedness. 
One of the original contributions of the proposed programme is its inclusion of tradition populaire, according to which communication will be made not only with residents in the disaster area but also with inbound visitors. The proposed disaster prevention training programme based on ICT can support disaster areas through educating inbound visitors, enhancing both citizen and visitor understanding of disastrous outcomes and increasing preparedness for disasters.

\subsection{Further research planning}

The critical topic of how to evaluate and improve trainees' feedback should be measured and reflected upon to create a revised version of the training programme. The review and proposition of a more robust training programme framework with key factors should also be conducted using a quantitative approach, following the experimental phase of the study. As a further research plan, it will be crucial to validate the usefulness of the proposed action plan containing key factors. In doing so, a preventative training programme contributing both to enhancing citizens' disaster preparedness and revitalising communities with inbound impact can be realised.

\section{References}

[1] Tsuneyoshi, R. (2019). Safety and Disaster Prevention Education as Education for Life. Tokkatsu: The Japanese Educational Model Of Holistic Education, 69. 
[2] Magnussen, L. I., Carlström, E., Sørensen, J. L., Torgersen, G. E., Hagenes, E. F., \& Kristiansen, E. (2018). Learning and usefulness stemming from collaboration in a maritime crisis management exercise in Northern Norway. Disaster Prevention and Management, 27(1), 129-140.

[3] Sun, Y., Yamori, K., Tanisawa, R., \& Kondo, S. (2013). Consciousness of disaster risk and tsunami evacuation: a questionnaire survey in Okitsu, Kochi Prefecture. Journal of Natural Disaster Science, 34(2), 127-141.

[4] Hatayama, M., \& Nakai, F. (2020). Using Computer Simulation for Effective Tsunami Risk Communication. In Disaster Risk Communication (pp. 39-50). Springer, Singapore.

[5] Hangartner, R. B., Totura, C. M. W., Labouliere, C. D., Gryglewicz, K., \& Karver, M. S. (2019). Benchmarking the "Question, Persuade, Refer" program against evaluations of established suicide prevention gatekeeper trainings. Suicide and Life - Threatening Behavior, 49(2), 353-370.

[6] Sun, Y. (2020). New Approaches Toward Tsunami Risk Preparedness in Japan. In Disaster Risk Communication (pp. 17-38). Springer, Singapore.

[7] Goltz, J. D. (2017). Tsunami Tendenko: A Sociological Critique. Natural Hazards Review, 18(4), 04017011). 
[8] Thompson, C. S. (2019). Folk spirituality, ghosts, and tsunami death-mitigation in Iwate, Japan: a local take on the legends of tōno-story 99. Asian Anthropology, 18(2), 91109.

[9] Kawakami, S., Oe, H., \& Yamaoka, Y. (2020). An exploratory study of how to increase citizens' preparedness for natural disasters using e-learning system: From Japanese experiences.

[10] Kodama, S. (2015). Tsunami-tendenko and morality in disasters. Journal of medical ethics, 41(5), 361-363.

[11] Maruyama, H. (2018). Learning Opportunities Since the Great Earthquake of 2011. In Japanese Education in a Global Age (pp. 195-210). Springer, Singapore.

[12]Enshu, H. (2014). How Do We Estimate Tsunami Tendenko, Local Lore of Disasters?, Community, university, and culture, Annual report of the Community Outreach Center, Graduate School of Humanities, Kobe University, 6, 20-35.

[13]Kamaishi City (2016). Kamaishi City Testimony / Record Collection "Memory of 3.11 I Want to Tell", Available at: https://www.city.kamaishi.iwate.jp/docs/2018091800049/ [Accessed 15 October 2020]

[14] Maeda, K. \& Hashimoto, H. (2020). Exploring the potential of the practice of Inochitendenko in current disaster prevention education: The interaction effect of teachers' 
attitudes toward disaster prevention education and the organizational climate in schools, Japanese Journal of Social Psychology, 35(3), 91-98.

[15] Ministry of Education, Culture, Sports, Science and Technology (2013). Reference materials for school disaster prevention Development of disaster prevention education that fosters "zest for life", Available at: https://www.mext.go.jp/component/a_menu/education/detail/__icsFiles/afieldfile/2018/1 2/25/1334780_01.pdf [Accessed 15 October 2020]

[16]Oe, H. and Yamaoka, Y. (2020), A study of social preparedness for natural disasters with communication and practices: Community as a safety net, The Open University of Japan (Research Office working Paper Series: 6-01-2020).

[17] Mikami, T., Shibayama, T., Esteban, M., \& Aránguiz, R. (2020). Comparative Analysis of Triggers for Evacuation during Recent Tsunami Events. Natural Hazards Review, 21(3), 04020022.

[18] Paton, D. (2019). Disaster risk reduction: Psychological perspectives on preparedness. Australian journal of psychology, 71(4), 327-341.

[19] Mitsuhara, H. (2018). The present and future of ICT-based disaster education system, Journal of Japanese Society for Information and Systems in Education, 35(2), 66-80. 
[20] Yanagawa, Y., Takeuchi, I., Jitsuiki, K., Yoshizawa, T., Ishikawa, K., Omori, K. \& Osaka, H. (2016).Disaster Imagination Game at Izunokuni City for preparedness for a huge Nankai Trough earthquake, Scholars Journal of Applied Medical Sciences, 4(6D):2129-2132.

[21] Guo, Y., \& Li, Y. (2016). Getting ready for mega disasters: the role of past experience in changing disaster consciousness. Disaster Prevention and Management, $25(4), 492-505$

[22] Palen, L., \& Liu, S. B. (2007). Citizen communications in crisis: anticipating a future of ICT-supported public participation. In Proceedings of the SIGCHI conference on Human factors in computing systems (pp. 727-736).

[23] Skinner, J. (2018). "The Smoke of an Eruption and the Dust of an Earthquake": Dark Tourism, the Sublime, and the Re-animation of the Disaster Location. In The Palgrave handbook of dark tourism studies (pp. 125-150). Palgrave Macmillan, London.

[24] Stone, P. (2013). Dark tourism scholarship: A critical review. International Journal of Culture, Tourism and Hospitality Research, 7(3), 307-318.

[25] Hooper, G., \& Lennon, J. J. (Eds.). (2016). Dark tourism: Practice and interpretation. Routledge. 
[26] Denshoroad organization (2019). 3.11 Traditional Road, Available at: https://www.311densho.or.jp/ [Accessed 16 October 2020]

[27] Sasaki, K., Yamamoto, K., and SYamamoto, S. (2018). Current Issues and Possibilities of Dark Tourism in the Consciousness of Visitors to Ishinomaki City after the Great East Japan Earthquake, Environmental Information Science, 32, 161-166.

[28] Oe, H. (2019a), Natural disaster \& Information gap: How to support disabled people with ICT applications, The Open University of Japan (Research Office Working Paper Series:16-12-2019)

[29] Oe, H. (2011a), A study on the learning process of sustainable community design: from the process of etude based on the case method approach, Departmental Bulletin Paper, Yokohama National University, 32(1), 99-112.

[30] Lovreglio, R., \& Kinateder, M. (2020). Augmented reality for pedestrian evacuation research: promises and limitations. Safety science, 128, 104750 (In-printing)

[31] Macchione, F., Costabile, P., Costanzo, C., \& De Santis, R. (2019). Moving to 3-D flood hazard maps for enhancing risk communication. Environmental Modelling \& Software, 111, 510-522.

[32] Solinska-Nowak, A., Magnuszewski, P., Curl, M., French, A., Keating, A., Mochizuki, J., Liu, W., Mechlera, R., Kulakowskab, M., \& Jarzabek, L. (2018). An overview of 
serious games for disaster risk management-Prospects and limitations for informing actions to arrest increasing risk. International journal of disaster risk reduction, 31, 10131029.

[33] Scharmer, C. Otto (2007). Theory U: Leading from the Future as it Emerges. The Society for Organizational Learning, Cambridge, USA.

[34] Oe, H. (2011b), A study of risk communication and interactive understanding, Departmental Bulletin paper, 32 (3/4)37-53.

[35] Oe, H. (2019b), ITU standards for disaster relief, network resilience and recovery: Our discussion agenda, The Open University of Japan (Research Office Working Paper Series:16-09-2019).

[36] Tetnowski, J. (2015). Qualitative case study research design. Perspectives on Fluency and Fluency Disorders, 25(1), 39-45.

[37] Edmonds, W. M. (2019). Snowballing: A Qualitative Study Using Snowball Sampling. SAGE Publications Ltd.

[38] Jentoft, N., \& Olsen, T. S. (2019). Against the flow in data collection: How data triangulation combined with a 'slow' interview technique enriches data. Qualitative Social Work, 18(2), 179-193. 
[39] Kern, F.G. (2018). The trials and tribulations of applied triangulation: Weighing different data sources. Journal of Mixed Methods Research, 12(2), 66-181.

[40] Auerbach, C., \& Silverstein, L. B., 2003. Qualitative data: An introduction to coding and analysis (Vol. 21). NYU Press.

\section{Figures}

How to fill the gap between the reality and 'possible' disasters

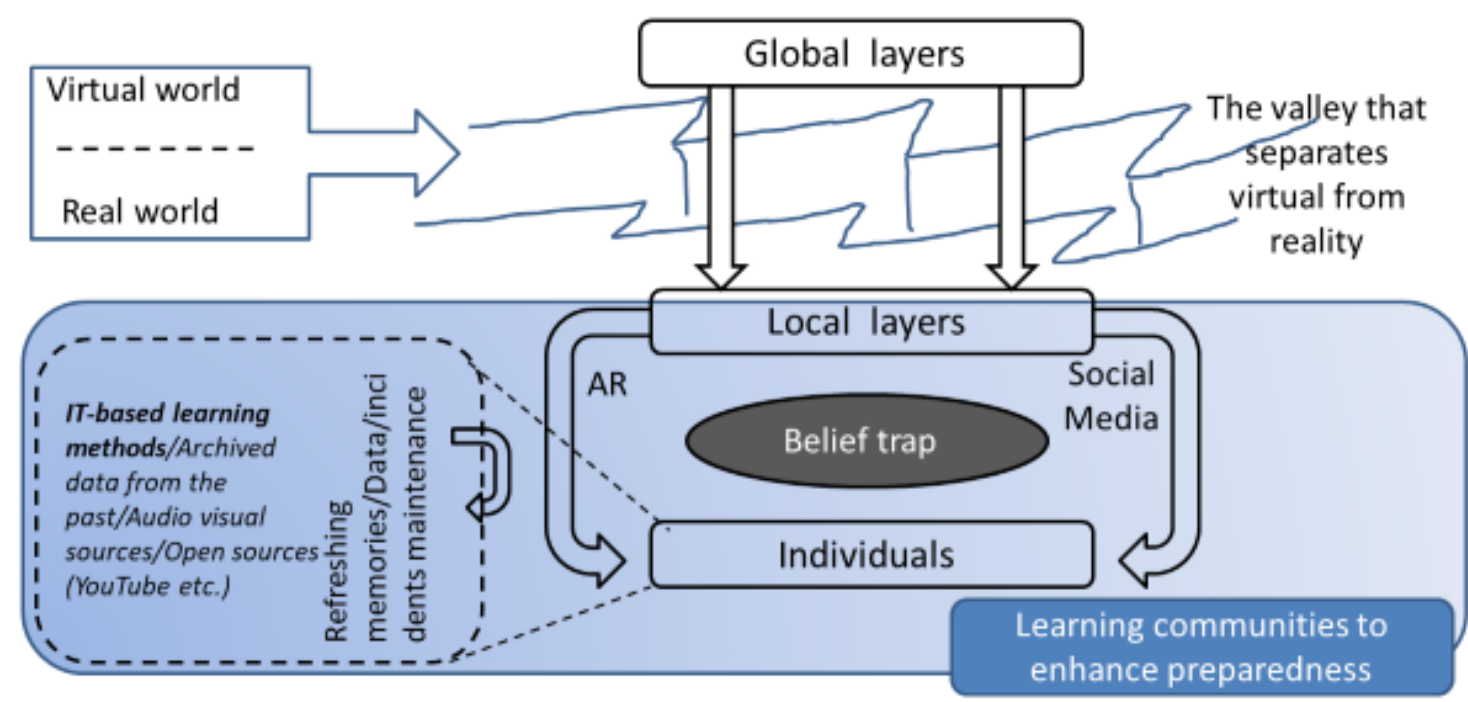

Figure 1: Virtual and real word perception gap model (Inspired by Mitsuhara (2018) and arranged by the authors) 


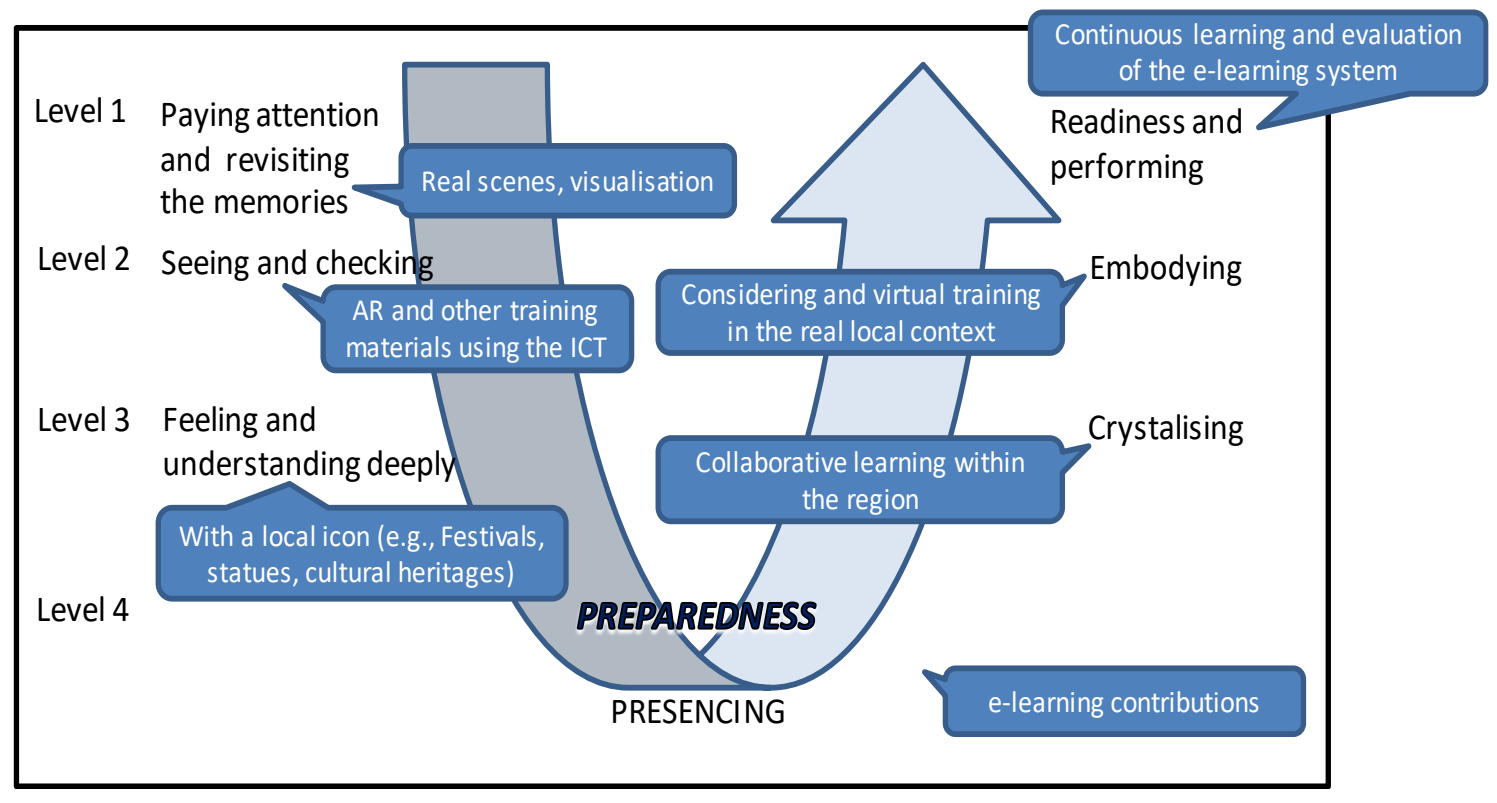

Figure 2: A steps to enhance preparedness and readiness based on the e-

learning training (Inspired by Scharmer (2017) and arranged by the authors)

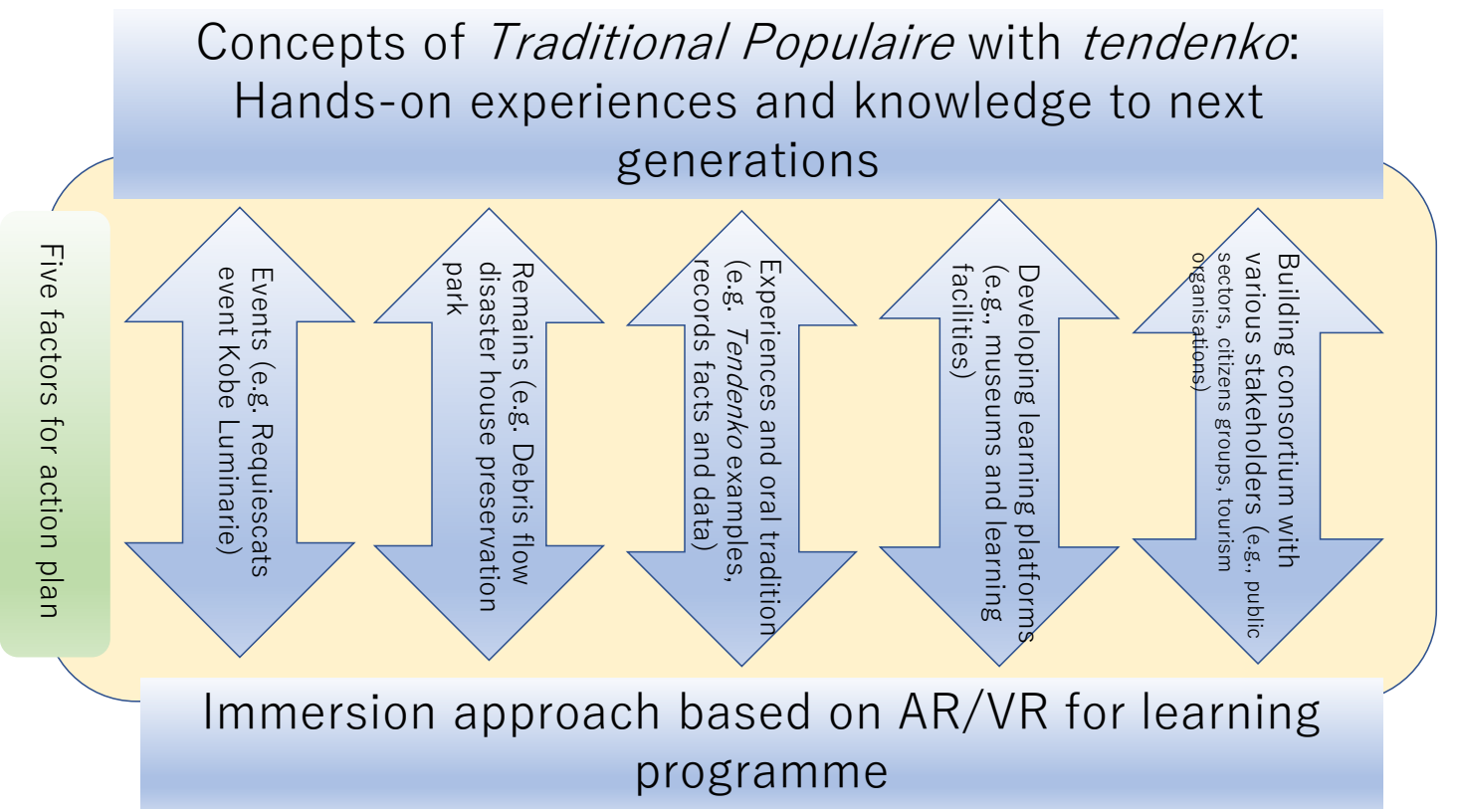

Figure 3: Action plan for five sources to design immersion programme based on Tradition Populaire with tendenko concept (Authors' collection) 